\title{
A novel nomogram containing acute radiation esophagitis predicting radiation pneumonitis in thoracic cancer receiving radiotherapy
}

Wenjie Tang ${ }^{1,2}$, Xiaolin $\mathrm{Li}^{2}$, Haining Yu ${ }^{3}$, Xiaoyang Yin ${ }^{1,2}$, Bing Zou ${ }^{2}$, Tingting Zhang ${ }^{4}$, Jinlong Chen ${ }^{4}$, Xindong Sun ${ }^{2}$, Naifu Liu, Jinming $Y u^{2}$ and Peng $X i^{2^{*}}$

\begin{abstract}
Background: Radiation-induced pneumonitis (RP) is a non-negligible and sometimes life-threatening complication among patients with thoracic radiation. We initially aimed to ascertain the predictive value of acute radiationinduced esophagitis (SARE, grade $\geq 2$ ) to symptomatic RP (SRP, grade $\geq 2$ ) among thoracic cancer patients receiving radiotherapy. Based on that, we established a novel nomogram model to provide individualized risk assessment for SRP.

Methods: Thoracic cancer patients who were treated with thoracic radiation from Jan 2018 to Jan 2019 in Shandong Cancer Hospital and Institute were enrolled prospectively. All patients were followed up during and after radiotherapy (RT) to observe the development of esophagitis as well as pneumonitis. Variables were analyzed by univariate and multivariate analysis using the logistic regression model, and a nomogram model was established to predict SRP by "R" version 3.6.0.

Results: A total of 123 patients were enrolled (64 esophageal cancer, 57 lung cancer and 2 mediastinal cancer) in this study prospectively. RP grades of 0, 1, 2, 3, 4 and 5 occurred in 29, 57, 31, 0, 3 and 3 patients, respectively. SRP appeared in 37 patients (30.1\%). In univariate analysis, SARE was shown to be a significant predictive factor for SRP $(P<0.001)$, with the sensitivity $91.9 \%$ and the negative predictive value $93.5 \%$. The incidence of SRP in different grades of ARE were as follows: Grade 0-1: 6.5\%; Grade 2: 36.9\%; Grade 3: 80.0\%; Grade 4: 100\%. Besides that, the dosimetric factors considering total lung mean dose, total lung V5, V20, ipsilateral lung mean dose, ipsilateral lung V5, and mean esophagus dose were correlated with SRP (all $P<0.05$ ) by univariate analysis. The incidence of SRP was significantly higher in patients whose symptoms of RP appeared early. SARE, mean esophagus dose and ipsilateral mean lung dose were still significant in multivariate analysis, and they were included to build a predictive nomogram model for SRP.

(Continued on next page)
\end{abstract}

\footnotetext{
*Correspondence: xiepengro@126.com

${ }^{2}$ Department of Radiation Oncology, Shandong Cancer Hospital and Institute,

Shandong First Medical University and Shandong Academy of Medical

Sciences, Jiyan Road 440, Jinan 250117, Shandong, China

Full list of author information is available at the end of the article
} 
(Continued from previous page)

Conclusions: As an early index that can reflect the tissue's radiosensitivity visually, SARE can be used as a predictor for SRP in patients receiving thoracic radiation. And the nomogram containing SARE may be fully applied in future's clinical work.

Keywords: Nomogram, Thoracic cancer, Acute radiation-induced esophagitis, Symptomatic radiation-induced pneumonitis, Radiotherapy

\section{Background}

Radiotherapy (RT) plays a crucial role in the treatment of thoracic cancer, which carries nontrivial risks of radiation-induced lung toxicity (RILT) at the same time [1]. Moreover, this toxicity may cause a significant decline in the quality of life and the survival time. And the commonest form of RILT is radiation-induced pneumonitis (RP). It is a dose-limiting complication for thoracic cancer patients undergoing RT [2]. The lung has been reported to be sensitive to the deleterious effects of ionizing radiation [1, 3]. Acute radiationinduced pneumonitis often occurs within 6 months after finishing of RT [1, 4], which can lead to pulmonary failure and even become life-threatening $[5,6]$. Besides, the radiation-induced lung injury is usually irreversible. Notably, the establishment of early RP predictors is a significant work for clinicians.

The pathogenetic process leading to RP is an integrated response to the complex organization of lung tissue, which include edema, epithelial degeneration and subsequent regeneration, invasion of alveoli by bronchial epithelium, endothelial sloughing, disruption of microvasculature, as well as atelectasis [2]. Radiation induced pulmonary damage can be varied and often long-lasting. Usually, it starts as a kind of exudative inflammation and end with scar formation which called lung fibrosis. That is the commonest end of radiation-induced lung damage [3] .

Previous studies have shown the importance of clinical characteristics, dosimetric parameters factors as well as laboratory indicators, such as pack-years, baseline pulmonary function, a history of lung resection, mean lung dose (MLD), total or ipsilateral lung volume receiving more than 2000 cGy (V20), total lung V10, total lung V13, ipsilateral lung V5, interleukin-8, recombinant human eotaxin-2, recombinant human eotaxin-22, recombinant human eotaxin-17 and so on [6-13]. In addition, genetic variants of pulmonary surfactant-associated glycoprotein $\mathrm{D}$, homeodomain interacting protein kinase 2 and interleukin-4 were also reported to be associated with RP development [14-16]. And the combination of chemotherapy has also been implicated in increasing chances of developing RP [10, 17, 18].

Although numbers of predictors have been reported to be related with $\mathrm{RP}$, few of them can be truly applied clinically. There is still a lack of consensus on reliable predictors for clinicians. More so, an accurate RP predictive model with superior clinical utility is urgently needed. By a long term of observation, we observed a novel and interesting phenomenon that patients with symptomatic radiation-induced pneumonitis (grade $\geq 2$, SRP) often suffered severe acute radiation-induced esophagitis (grade $\geq 2$, SARE) during RT. This study aimed for exploring the predictive value of SARE to SRP, and establishing a visually nomogram model for SRP among patients undergoing thoracic RT.

\section{Methods \\ Patients cohort}

From Jan 2018 to Jan 2019, patients with thoracic malignancies such as lung cancer, esophageal cancer and some mediastinal malignant tumors were included in this study prospectively. All patients were treated with thoracic RT with or without concurrent chemotherapy. In order to explore the relation between pneumonitis and esophagitis, we selected patients whose esophagus and lung tissue were both exposed to radiation. As a result, most of the included patients were central bronchogenic carcinoma and esophagus cancer with mediastinal lymph node metastasis. The type of RT was determined by both the patient and the clinician. Most of them tolerated a total dose $\geq 5000$ cGy. The inclusion criteria contained: (1) Receipt of thoracic radiotherapy; (2) Karnofsky Performance Status $\geq 70$; (3) Age $\geq 18$; (4) Ipsilateral lung volume receiving 500 cGy (V5) $\geq 20 \%$ and maximum esophagus dose $\geq 4500 \mathrm{cGy}$ (in order to select patients whose esophagus and lung tissue were both exposed to radiation). The exclusion criteria included: (1) Previous history of thoracic RT; (2) Receipt of stereotactic body RT; (3) Patients with a life expectancy of less than 6 months (because they might not benefit from local radiation and might not be assessable for late lung toxicity); (4) Severe complications such as coronary heart disease, $\geq \operatorname{grad} 3$ hypertension and $\geq \operatorname{grad} 3$ chronic obstructive pulmonary diseases; The fasting bloodglucose of patients with diabetes need to be controlled $\leq 7 \mathrm{mmol} / \mathrm{L}$. (5) Patients that didn't come back to the hospital to take a chest computed tomography (CT) reexamination among the 6 months after RT; (6) Patients that failed to be contacted. No restrictions were placed 
on either the use of cytotoxic chemotherapy or the stage of disease. We seek for appropriate patients clinically, then observe and record the time, RTOG grade and duration of esophagitis symptoms during radiotherapy. All patients were followed up every month in the first 6 months after RT until the RP occurred.

\section{Feature definition and endpoints}

We analyzed 31 continuous and categorical variables in this study. The continuous features were age, smoking pack-years, total radiation dose, number of radiation fractions, radiation fraction size, some indicators of pulmonary function (forced vital capacity FVC, forced expiratory volume in the first second FEV1, peak expiratory flow PEF, etc.), and some peripheral blood indicators before RT (erythrocyte count, white blood cell count, neutrophil count and lymphocyte count). We also collected the lowest hematology index during RT (white blood cell count, neutrophil count and lymphocyte count). We obtained some dosimetric parameters from dose-volume histogram (DVH) such as total lung V5, ipsilateral lung V20, mean esophagus dose, etc. For esophageal carcinoma or mediastinal tumor, ipsilateral lung refers to the side that received more radiation dose in RT. The time that pneumonitis symptoms appear after RT were included. The categorical features were gender, smoking status, SARE, esophagitis duration time after RT, etc. All data, including clinical parameters and the grade of RP/ARE were collected prospectively. ARE was graded in accordance with the Radiation Therapy Oncology Group (RTOG) acute radiation injury grading criteria. The primary endpoint of this study was SRP, defined in the Common Terminology Criteria for Adverse Events (CTCAE) version 5.0 [19] published by the National Cancer Institute (Table 1). The choice of RT pattern was jointly formulated by the clinicians, physicists as well as patients themselves, and the pulmonary function test was determined by the individual condition of every patient. The assessment of RP and
Table 2 Patient characteristics ( $N=123)$

\begin{tabular}{|c|c|}
\hline Variables & Cohort, No. (\%) \\
\hline Median age & 64 (range $25 \sim 81$ ) \\
\hline \multicolumn{2}{|l|}{ Sex } \\
\hline Male & $94(76.40)$ \\
\hline Female & $29(23.60)$ \\
\hline \multicolumn{2}{|l|}{ Smoking status } \\
\hline No smoking & $52(42.30)$ \\
\hline Current & $31(25.20)$ \\
\hline Former & $40(32.50)$ \\
\hline \multicolumn{2}{|l|}{ Tumor type } \\
\hline Lung cancer & $57(46.30)$ \\
\hline Esophageal cancer & $64(52.00)$ \\
\hline Mediastinal malignant fibrous adenoma & $2(1.60)$ \\
\hline \multicolumn{2}{|l|}{ RT modality } \\
\hline IMRT & 103(83.74) \\
\hline 3D-CRT & $15(12.20)$ \\
\hline TOMO & $5(4.06)$ \\
\hline \multicolumn{2}{|l|}{ Total prescribed dose (cGy) } \\
\hline$\geq 6000$ & $64(52.03)$ \\
\hline $5000 \sim 6000$ & $24(19.51)$ \\
\hline$\leq 5000$ & $35(28.46)$ \\
\hline \multicolumn{2}{|l|}{ ARE } \\
\hline Grade 0-1 & $46(37.40)$ \\
\hline Grade 2-5 & $77(62.60)$ \\
\hline \multicolumn{2}{|l|}{$\mathrm{RP}$} \\
\hline Grade 0-1 & $86(69.90)$ \\
\hline Grade $2-5$ & $37(30.10)$ \\
\hline
\end{tabular}

Abbreviations: $O R$ odds ratio, $\mathrm{Cl}$ confidence interval, $R T$ radiotherapy, IMRT intensity-modulated radiotherapy. 3D-CRT 3D-conformal radiotherapy, TOMO tomotherapy; $A R E$ acute radiation-induced esophagitis, $R P$ radiation-induced pneumonitis

Table 1 The assessment criteria of RP and ARE in this research

\begin{tabular}{lll}
\hline Grade & Radiation Therapy Oncology Group grading for ARE & $\begin{array}{l}\text { Common Terminology Criteria for Adverse Events } \\
\text { (CTCAE) v5.0 for RP }\end{array}$ \\
\hline 0 & None & None \\
1 & $\begin{array}{l}\text { Mild dysphagia or odynophagia may require topical anesthetic or non-narcotic } \\
\text { analgesics/may require soft diet }\end{array}$ & $\begin{array}{l}\text { Asymptomatic; clinical or diagnostic observations only; } \\
\text { intervention not indicated }\end{array}$ \\
2 & $\begin{array}{l}\text { Moderate dysphagia or odynophagia may require narcotic analgesics/may } \\
\text { require puree or liquid diet }\end{array}$ & $\begin{array}{l}\text { Symptomatic; medical intervention indicated; limiting } \\
\text { instrumental ADL }\end{array}$ \\
3 & $\begin{array}{l}\text { Severe dysphagia or odynophagia with dehydration or weight loss > 15\% from } \\
\text { pretreatment baseline/requiring N-G feeding tube, iv. fluids or hyperalimentation }\end{array}$ & Severe symptoms; limiting self-care ADL; oxygen indicated \\
4 & Complete obstruction, ulceration, perforation, fistula & $\begin{array}{l}\text { Life-threatening respiratory compromise; urgent } \\
\text { intervention indicated (e.g., tracheotomy or intubation) }\end{array}$ \\
5 & Death
\end{tabular}




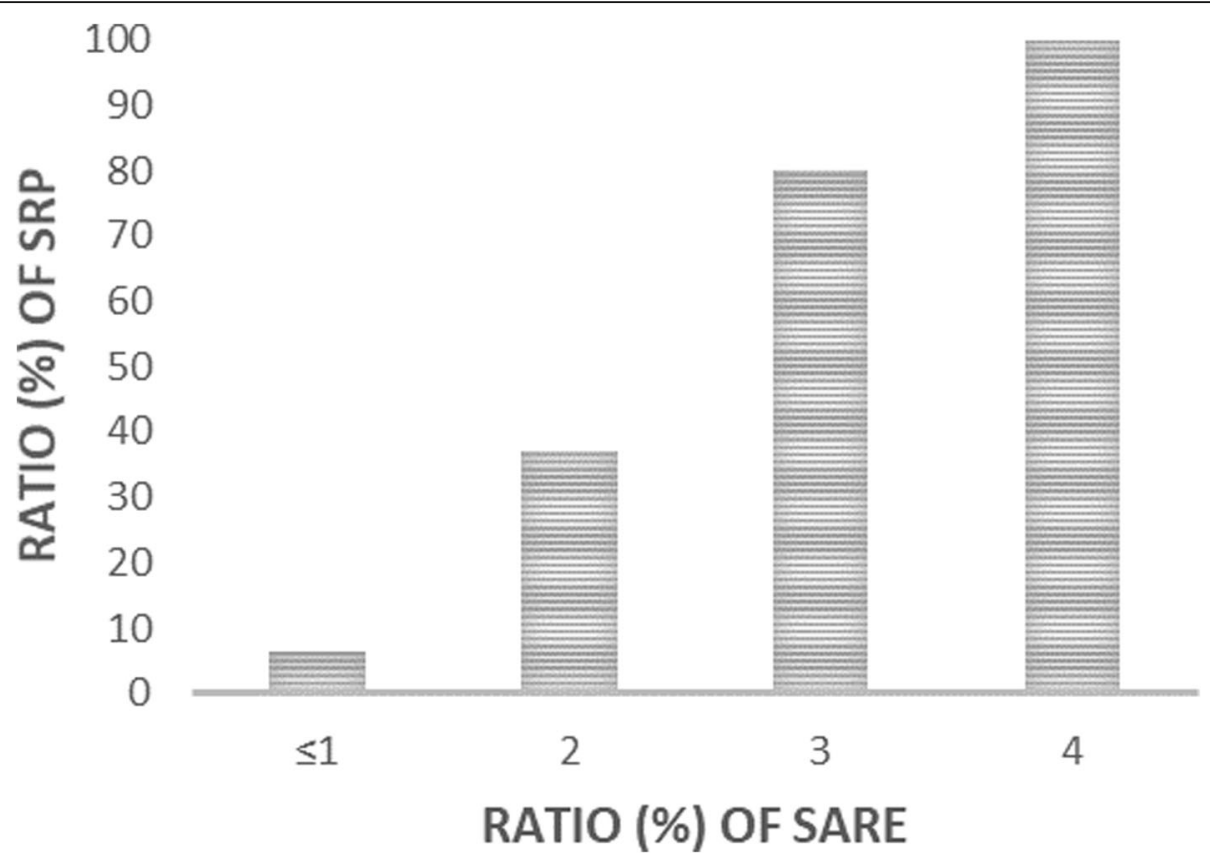

Fig. 1 Incidence of SRP in different grades of ARE population. Abbreviations: ARE: acute radiation-induced esophagitis; SRP: severe radiation-induced pneumonitis

SARE was diagnosed by 2 radiation oncologists or pulmonologists based on patients' clinical symptoms and the range of radiographic infiltration within the radiation field during the first 12 months after radiation.

\section{Statistics}

Univariate and multivariate logistic regression analysis were performed to explore features associated with SRP. We also used the Gamma rank correlation coefficient to further confirm the relation between SARE and SRP. All statistical tests were two-tailed, with a $P$ value less than 0.05 considered to be statistically significant. Receiver operating characteristic (ROC) curve analysis was used to confirm optimal cut points for variables identified as influencing RP in this analysis. The variables were subjected to multivariate analysis only when a significant difference $(P<0.05)$ was computed in the univariate analysis. Cumulative incidence of SRP was calculated from the end of RT to the date of SRP occurrence or to the date of last follow-up, patients lost to follow-up were censored. All statistical analyses were performed using the SPSS version 25.0. And the nomogram model was established by the package of rms in " $R$ " version 3.6.0.

Table 3 Analysis of factors associated with Grade $\geq 2$ RP

\begin{tabular}{|c|c|c|c|c|c|c|c|}
\hline Variables & OR & $\begin{array}{l}\text { Univariate } \\
P \text {-value }\end{array}$ & $\begin{array}{l}\text { analysis } \\
95 \% \mathrm{Cl}\end{array}$ & AUC & OR & $\begin{array}{l}\text { Multivariate } \\
P \text {-value }\end{array}$ & $\begin{array}{l}\text { analysis } \\
95 \% \mathrm{Cl}\end{array}$ \\
\hline$\overline{\text { SARE }}$ & 11.333 & 0.000 & $3.235-39.710$ & 0.778 & 16.763 & 0.000 & $3.638-77.230$ \\
\hline TLMLD & 1.001 & 0.024 & $1.000-1.002$ & 0.615 & & & \\
\hline TLV5 & 1.034 & 0.035 & $1.002-1.067$ & 0.586 & & & \\
\hline TLV20 & 1.063 & 0.035 & $1.004-1.124$ & 0.590 & & & \\
\hline ILMLD (>1186.78 cGy) & 3.955 & 0.018 & $1.270-12.317$ & 0.610 & 3.557 & 0.048 & $1.013-12.495$ \\
\hline ILV5 (>55.65\%) & 2.517 & 0.034 & $1.073-5.905$ & 0.595 & & & \\
\hline MED & 1.000 & 0.012 & $1.000-1.001$ & 0.641 & 1.000 & 0.044 & $1.000-1.001$ \\
\hline TIME & 0.986 & 0.001 & $0.978-0.994$ & 0.844 & & & \\
\hline
\end{tabular}

Abbreviations: $O R$ odds ratio, $C l$ confidence interval, SARE severe acute radiation-induced esophagitis, $T L$ total lung, IL ipsilateral lung, $M L D$ mean lung dose, $M E D$ mean esophagus dose, Time the time that pneumonia symptoms appear, AUC areas under the curve 


\section{Results}

\section{Patients' characteristics}

From Jan 2018 to Jan 2019, a total of 123 patients receiving thoracic RT were included prospectively in this study, which contained 57 (46.3\%) lung cancer patients, 64 (52.0\%) esophageal cancer patients and 2 (1.6\%) mediastinal malignant fibrous adenoma patients. The median age was 64 (range $25 \sim 81$ ) years old, and 94 (76.4\%) patients were male. Most patients (103, 83.7\%) received intensity-modulated radiation therapy (IMRT) in the study, 15 (12.2\%) patients received 3D-conformal RT (3D-CRT), and 5 (4.1\%) patients received tomotherapy (TOMO). The median dose of RT was $6000 \mathrm{cGy}$, which ranged from 4100 cGy to 6600 cGy. ARE appeared in this cohort were as follows: Grade $0 \sim 1$ in 46 (37.4\%) patients, Grade $2 \sim 5$ in $77(62.6 \%)$ patients. At the end of the follow up (July 6, 2019), 37 patients suffered SRP, 3 of them $(8.1 \%)$ died of RT-related respiratory failure. And among those patients, 34 (91.9\%) patients developed SARE. Table 2 shows the details of this study and patients' basal characteristics. And the incidence of SRP in different grades of ARE is explained in Fig. 1.

\section{Univariate analysis}

In univariate logistic regression analysis, SARE was proved to be strongly correlated with SRP (OR 11.333, 95\%CI 3.235-39.710, $P<0.001)$. Furthermore, we explored the correlation between SARE and SRP by using the Gamma rank correlation coefficient (0.838, $P<0.001)$. The sensitivity, specificity, positive predictive value and negative predictive value of SARE to predict SRP were 91.9, 50.0, 44.2 and 93.5\%, respectively. The false negative rate was $8.1 \%$. The dosimetric factors considered total lung MLD (TLMLD, OR $1.001 P=0.024$ ), ipsilateral lung MLD (ILMLD, OR 3.955, $P=0.018$ ), total Lung V5 (TLV5, OR 1.034, $P=0.035)$, V20 (TLV20, OR 1.063, $P=0.035$ ), ipsilateral lung V5 (ILV5, OR 2.517, $P=0.034$ ), mean

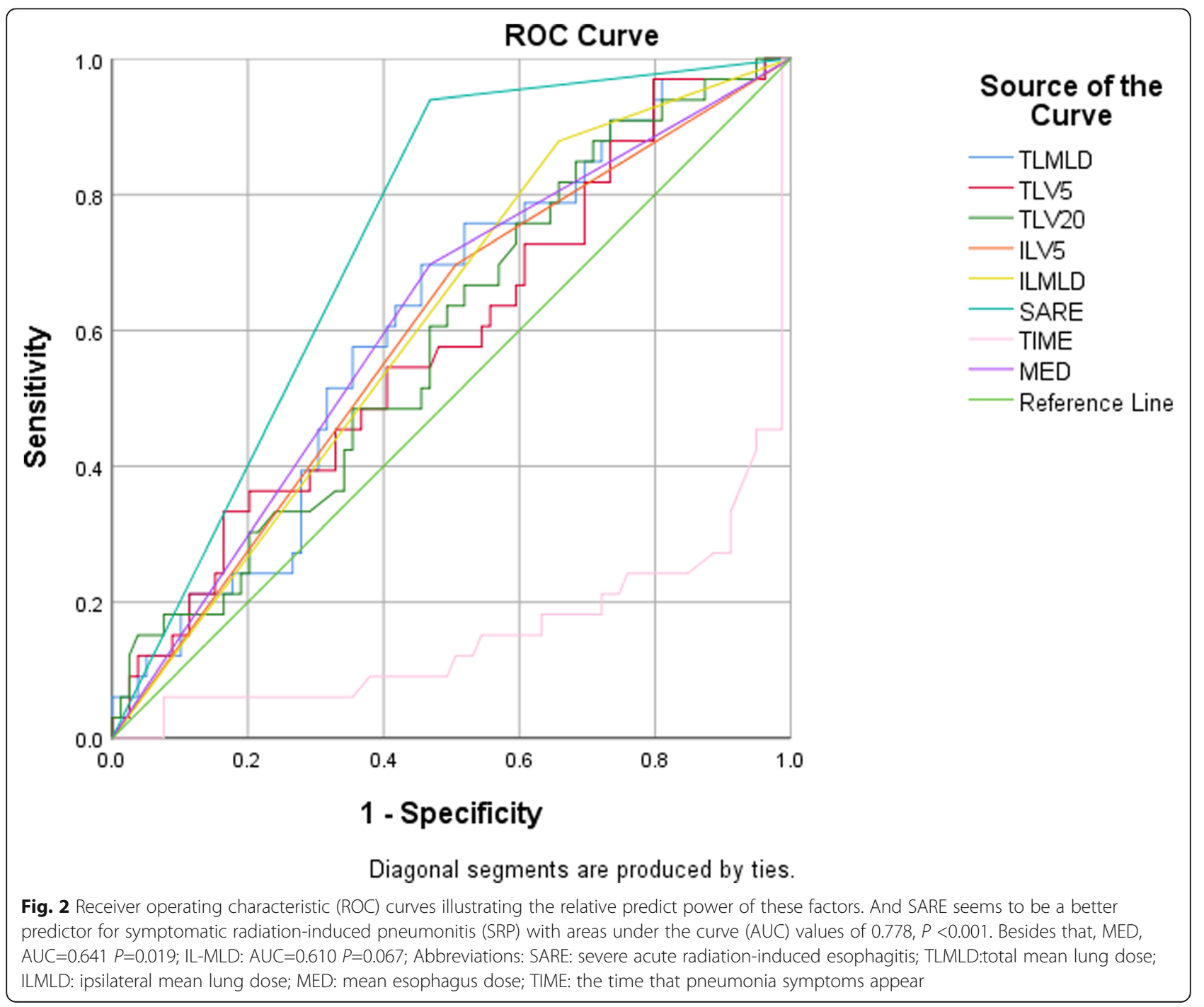


esophageal dose (MED, OR 1.000, $P=0.012$ ) correlated with SRP. Among these results, mean ipsilateral lung dose and ipsilateral lung V5 were nonsignificant when they expressed as continuous factors, then we used ROC analysis to determine the best cutoff points for mean ipsilateral lung dose (1186.78 cGy) and ipsilateral lung V5 (55.65\%). The areas under the curve (AUC) were 0.583 and 0.589 , respectively. And then, we found that when patients performed as mean ipsilateral lung dose $\geq 1186.78 \mathrm{cGy}$ and ipsilateral lung V5 $\geq 55.65 \%$, they might suffer a higher risk of RP. Besides that, the time that RP appeared after RT were also associated with the SRP (OR 0.986, $P=0.001$ ). No significant correlations were found for the peripheral blood information or pulmonary function index. Table 3 summarizes these above results. And the ROC curves illustrate the predictive value of SARE and other factors (Fig. 2).

\section{Multivariate analysis}

All above significant factors were included in the multivariate logistic regression analysis to find the most meaningful early predictors for SRP. And the multivariable logistic prediction model included three clinical variables (mean esophagus dose, mean ipsilateral lung dose and SARE), as shown in Table 3. SARE was shown to be the best independent risk predictor for the development of SRP (OR 16.763, 95\%CI 3.638-77.230, $P<0.001$ ).

\section{Nomogram model for SRP}

Finally, a visually predictive nomogram (Fig. 3) was formulated based on the results of multivariate logistic regression analysis. Fig. 4 demonstrates the area under the curve (AUC $=0.827, P<0.001$ ) of the data, and the predictive ability was estimated by AUC of ROC graphs. The ROC curve provides a visual representation of the sensitivity and specificity of measured parameters relative to RP risk. Bootstraps with 1000 resample were used for the validation of nomogram and calibration curve construction, and we can see a great match of the actual probability with the predicted probability in Fig. 5.

\section{Discussion}

Due to the significant morbidity and potential for mortality associated with RP, it has always been a tough nut to crack for the oncologists. As described above, previous researches have made great efforts to establish reliable predictors to guide clinicians in mitigating the radiation-induced lung toxicity. And the DVH parameters of the lung has been wildly applied in clinic, such as MLD and lung V20 [7-12]. However, these factors can't reflect the difference between individuals. In this study, we studied 31 parameters from 123 thoracic cancer patients enrolled prospectively. The results of this study demonstrated SARE, ILMLD (> 1186.78 cGy), MED were associated with SRP. And we further established an original nomogram model for symptomatic RP based on that.

To the best of our knowledge, it is the first time that SARE acts as a predictor for $\geq$ grade $2 \mathrm{RP}$. As we all know, both the radiation-induced pneumonitis and radiation-induced esophagitis are typical radiation toxicities among thoracic RT. However, no research has linked the two together before that. The symptoms of SARE include retrosternal pain, dysphagia and odynophagia. It is easy for an experienced radiation oncologist

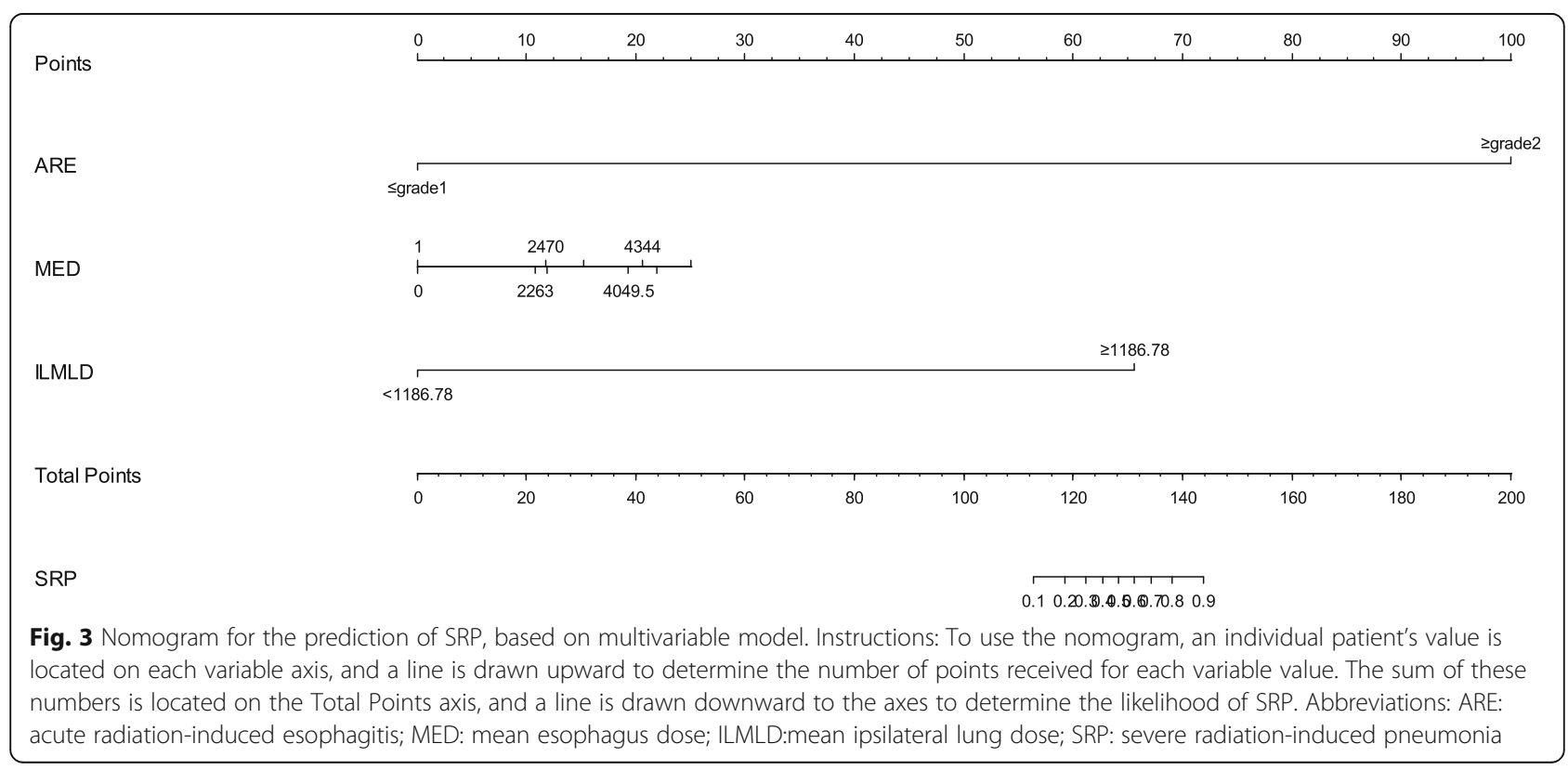




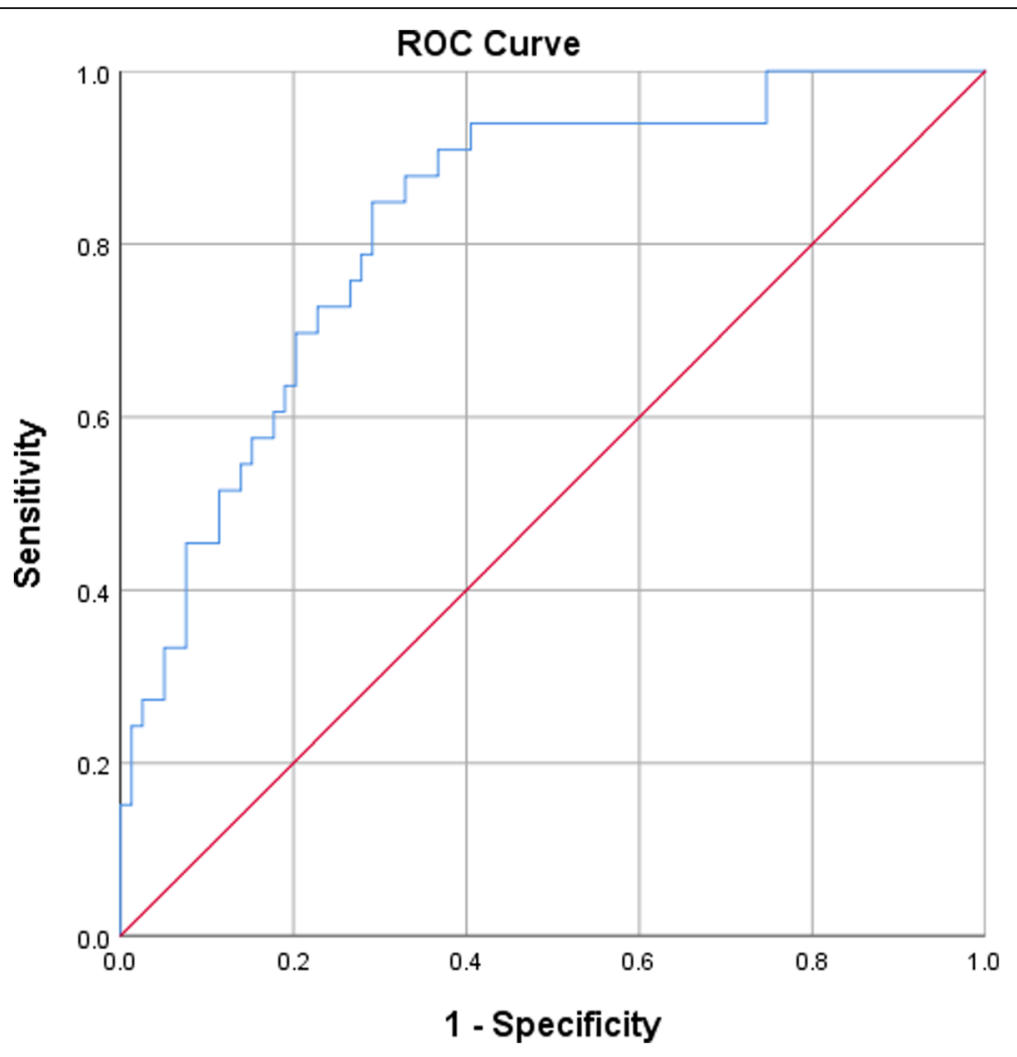

Fig. 4 ROC curve of the nomogram model for SRP: the area under the curve is 0.827 (95\% Cl: $0.746-0.908, P<0.001$ )

to estimate the severity of ARE by the patient's symptoms and physical signs during RT. SRP means that patients not only have changes on CT, but also have manifestations, such as cough, expectoration, dyspnea, etc. The assessment criteria of RP and ARE is explained in Table 1 in detail. By a long term of observation in clinic, we found a possible relation between these two inflammations and then designed a real-world study to explore the predictive value of SARE to SRP. Prior studies have explored numerous predictive factors for RP. Based on these results, we collected some of the clinical, laboratory and dosimetric parameters that we can obtain as much as possible. In order to better observe and record the initiation and development of patients' symptoms, we chose to collect information prospectively. The results highlighted the predictive value of SARE to SRP. And we think SARE may function as an easier and earlier signal for SRP in future's clinical work.

The high incidence of SRP was estimated to be at the range of $15 \sim 40 \%$ among patients with thoracic RT by previous studies [20], and the incidence in our study (30.8\%) was consistent with it. The mortality associated with RP was reported to be less than $2 \%$ [21], which was $1.6 \%$ in our study. The lethal ratio of RP was not extremely high, however, it did decrease the quality of life and led to poor prognosis [22, 23].
Moreover, the lung tissue may become more vulnerable to virus or bacterial infection. In this study, there was a 64-year-old esophageal cancer patient who died of RP related pulmonary infection. After only 10 fractions of RT, the old man suffered intractable grade 4 ARE. He felt extremely pain when swallowing and had difficulty in eating. The symptoms become worse and worse, then he can only drink a little water in the final stage of radiotherapy. The esophagoscopy found diffuse esophagus mucosal erosion, ulceration, and hemorrhage (Fig. 6a). Moreover, the symptoms continued for the subsequent treatment and even after RT. Then the pneumonitis symptoms appeared only 4 days after RT. Unfortunately, the man got infected with pneumocystis carinii during the following treatment of RP (Fig. $6 \mathrm{~b} \sim \mathrm{d}$ ) and died soon. This case reminds us that RP can not only reduce lung tolerance but increase the chance of severe pulmonary infection. If the doctor had realized the strong connection between SARE and SRP, he/she might modulate the radiotherapy plan timely. And the subsequent tragedy may be avoided by that.

In this real-word study, we set up both the esophagus and lung dose limits to the enrollment criteria to select those patients whose lung and esophagus tissue were both exposed to radiation. In another word, these 


\section{Calibration Curve}

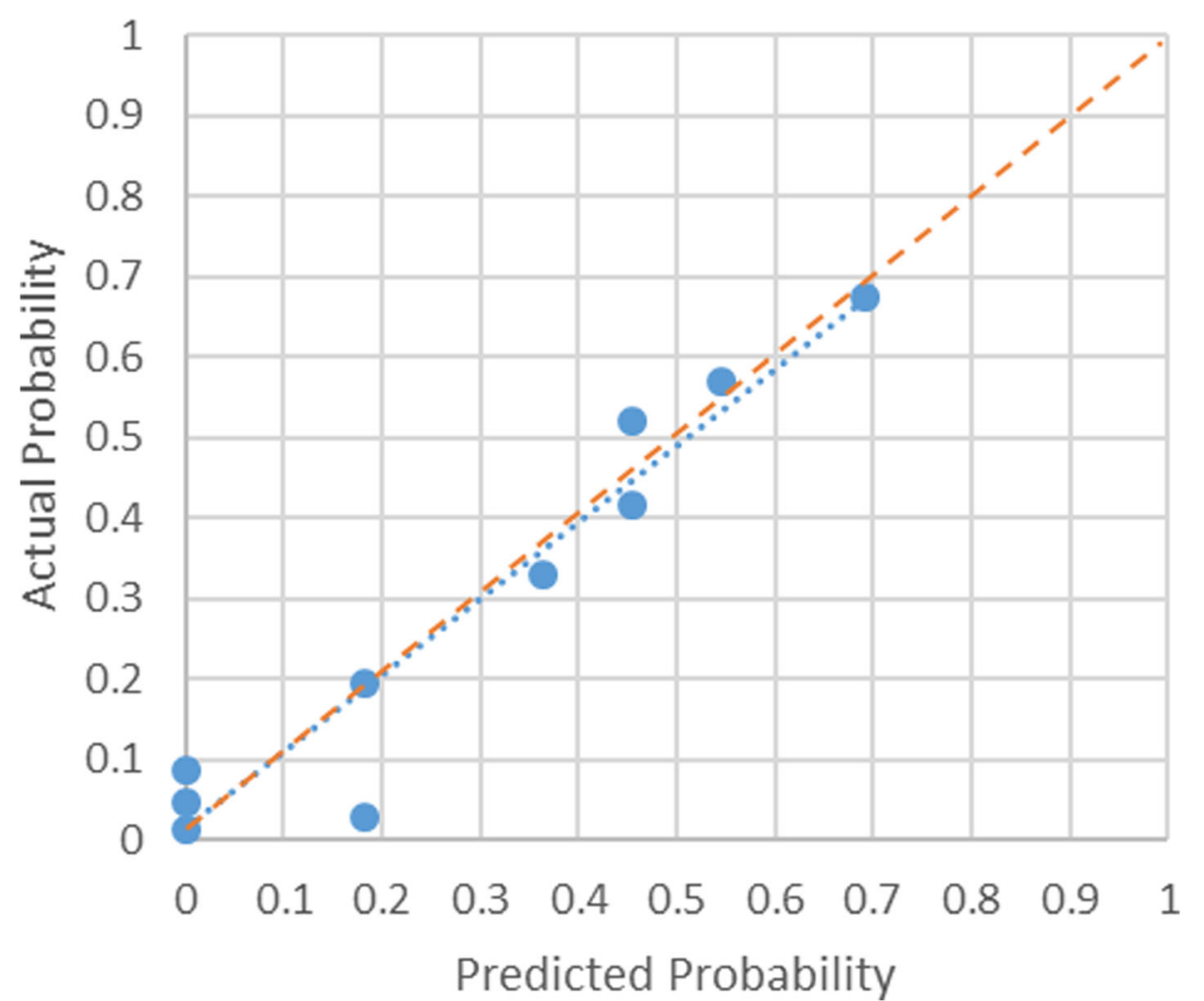

Fig. 5 Calibration Curve. Hosmer and Lemeshow Test: Chi-square $=10.975 P=0.203$ The predicted probability is almost the same as the actual probability, and the prediction error of the model is acceptable

patients have a highly likelihood of both two types of inflammation. The acute esophagus toxicity is regard as the response to the radiation exposure of esophageal mucosa. Patients often develop dysphagia and odynophagia, which may lead to appetite loss and weight loss $[24,25]$. These symptoms usually appear at 2 to 4 weeks during RT, and some even occur in the first 2 weeks. In addition to reducing the quality of life, SARE may cause treatment break, which is related to inferior survival results $[26,27]$. There is no specific cure for it, usually, ARE acts as a kind of self-limited complication. Symptoms can disappear in 2 to 4 weeks after the completion of RT gradually [27]. Our results showed SARE was associated with two esophagus-related dosimetric indicators, they were esophagus volume receiving more than 3000 cGy (V30) and the maximum esophagus dose. These two indicators showed a weak connection with SARE (OR: 1.000 and 1.010), which couldn't influence SRP. Thus, SARE could be used to reflect the sensitivity of individual's normal tissue towards the radiation directly. Patients whose radiation fields contain both the lungs and esophagus need to be more vigilant about the occurrence of SARE. If someone has developed SARE, great caution should be exercised on RP prevention. He/ she should pay more attention to keep warm during and after RT to avoid catching cold. And the patient should take timely chest CT to determine the lung condition and change the patient's treatment plan if necessary.

Numerous lung related DVH parameters for RP have been widely verified in previous studies, such as total or ipsilateral lung V5, V10, V13 and V20 [6-14]. Furthermore, the dosimetric parameters of esophagus, which may have not been served as predictors for RP, were also considered in this study. And we set enrollment limits on ILV5 and MED to reduce the bias of individual dose difference among the lung and esophagus, in order to avoid their affection on RP. We collected the parameters of mean esophagus dose and esophagus V30. We found that the MED made sense both in the univariate analysis and multivariate analysis. We admitted its value as a significant predictor for SRP, but that didn't mean it was one of the causes of RP. It served as an indirect indicator that could reflect the distribution and volume of pulmonary disease surrounding the esophagus, which eventually would be reflected in the pulmonary dosimetric outcomes. And previous study had already found that 

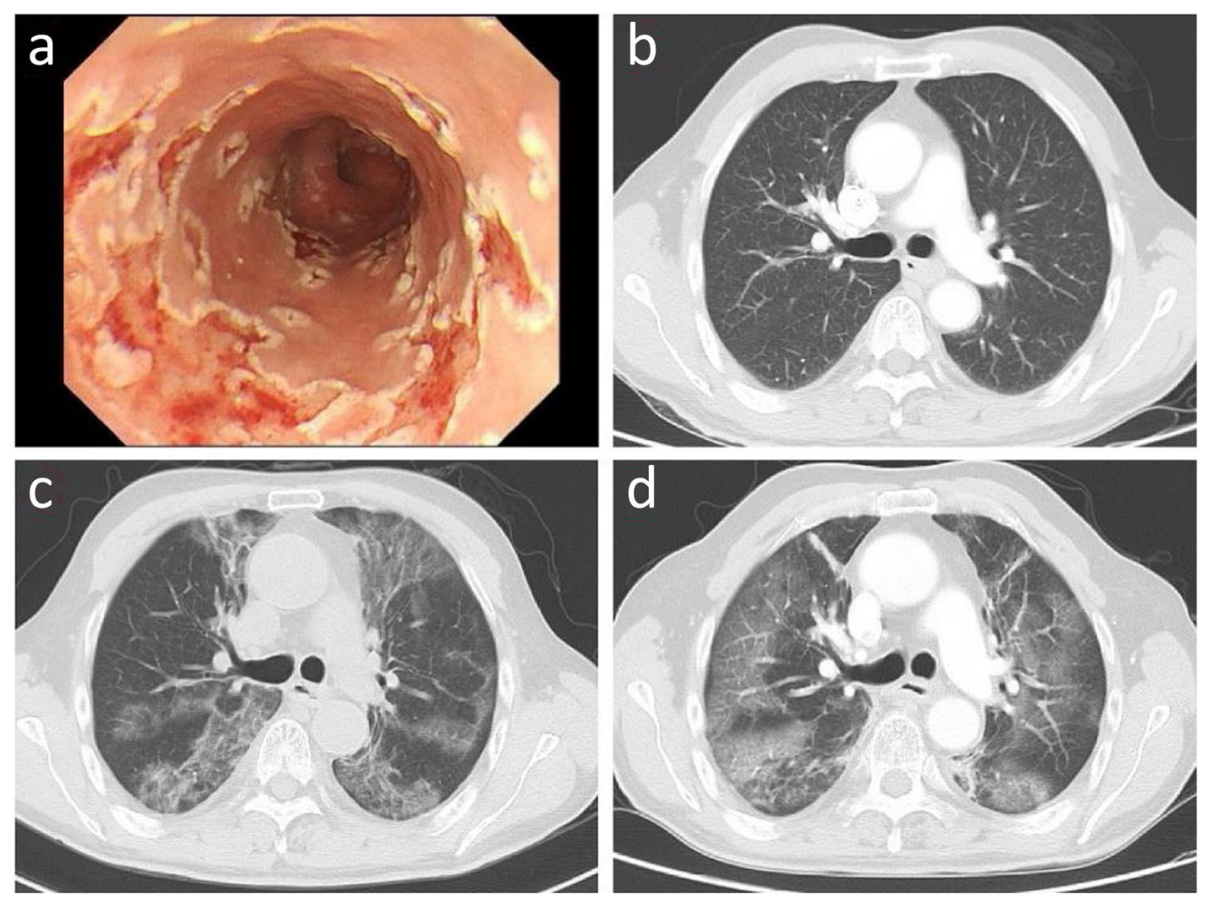

Fig. 6 This 64-year-old esophageal cancer patient suffered grade 4 severe acute radiation-induced esophagitis (SARE) after only 10 fractions of radiotherapy. And he developed symptomatic radiation-induced pneumonitis (SRP) only 4 days after radiotherapy (RT) and then got infected of pneumocystis carinii during RT treatment. a: Endoscopy reveals diffuse mucosal erosion, sloughing, ulceration, and hemorrhage, and the endoscopic secretion culture revealed the fungal infection. b: Computed tomography (CT) image before radiotherapy; $\mathbf{c}$ : CT image that 6 days after radiotherapy; $\mathbf{d}$ : $C T$ image that got infected of pneumocystis carinii during RP treatment.

esophagus dosimetric indices were positively correlated with lung dosimetric indices [7].

There are several possible reasons that may explain the predictive value of SARE to SRP. First of all, SARE can reflect the high esophageal radiation dose $[27,28]$, which further implicates the increase of radiation dose in the surrounding lung tissue. Secondly, the esophagus and lung are radiosensitive organs, and the early occurrence of SARE may further indicates the high radiosensitivity of the body tissue. More than this, Previous studies have confirmed multiple inflammatory cytokines were closely related to SARE as well as SRP [10, 27, 29, 30], so it may be a signal of activation of the body's systemic inflammatory system. However, more basic experiments are needed to better explain the relation.

Although we have obtained a considerable relation between SARE and SRP from 123 patients, it is far away to get a convincing conclusion. The main limitation of this study is the relatively small number of patients. More so, we validated the nomogram model internally by Bootstraps with 1000 resample instead of an external database. In addition, some more fundamental research toward the common nature of these two inflammations are needed to finally prove it.

\section{Conclusion}

A possible relation between SARE and SRP was demonstrated for the first time, especially for patients with central bronchogenic carcinoma and esophageal cancer. Compared with previously reported predictors, SARE can reflect the tissue' radiosensitivity visually. And SARE is easy to get assessed and further applied in clinic. The nomogram model containing SARE may assist the clinicians in identifying patients at great risk of SRP and guiding personalized radiation dose prescription or surveillance decisions. We hope this relation can be further confirmed by validation studies in the future.

\section{Abbreviations}

RT: Radiotherapy; RILT: Radiation-induced lung toxicity; RP: Radiation-induced pneumonitis; MLD: Mean lung dose; V20: Volume receiving more than 2000 cGy; SRP: Symptomatic radiation-induced pneumonitis; SARE: Severe acute radiation-induced esophagitis; FVC: Forced vital capacity; FEV1: Forced expiratory volume in the first second; PEF: Peak expiratory flow; DVH: Dosevolume histogram; RTOG: Radiation Therapy Oncology Group; CTCAE: Common Terminology Criteria for Adverse Events; ROC: Receiver operating characteristic; IMRT: Intensity-modulated radiation therapy; TOMO: Tomotherapy; 3D-CRT: 3D-conformal RT; IL: Ipsilateral lung; TL: Total lung; MED: Mean esophageal dose; AUC: Areas under the curve;

CT: Computed tomography

\section{Acknowledgements}

The authors wish to thank patients for supporting our work and thank editors as well as reviewers for reading the manuscript. The abstract was selected for presentation in the DIGITAL POSTER VIEWING Q\&A Session 
during the 2020 Annual ASTRO Meeting and it was included in the American Society for Radiation Oncology: https://www.redjournal.org/article/S0360-3 016(20)32747-4/fulltext.

\section{Authors' contributions}

Writing - original draft: WJT. Writing - review and editing: JMY, NFL and PX. Conceptualization: PX, XLL, HNY. Data collection: WJT, XYY, BZ, TTZ, JLC. Project administration: XDS, JMY, PX. All authors have read and approved the manuscript.

\section{Funding}

This project was supported by:

$\checkmark$ National Natural Science Foundation of China (Grant No. 81502667)

$\checkmark$ Key Research and Development Plan of Shandong, China (Grant No. 2016GSF201167)

$\checkmark$ Natural Science Foundation of Shandong, China (2019PH111)

The funders had role in the study design, data collection, article review and editing.

\section{Availability of data and materials}

The datasets used or analyzed during the current study are available from the corresponding author on reasonable request.

\section{Declarations}

\section{Ethics approval and consent to participate}

This study was approved by the Ethics Committee of Shandong Cancer Hospital and Institute, and written informed consents were obtained from all included individuals.

\section{Consent for publication}

Not Applicable.

\section{Competing interests}

The authors declare that they have no competing interests.

\section{Author details}

${ }^{1}$ Shandong First Medical University \& Shandong Academy of Medical Sciences, Jinan 250117, Shandong, China. ²Department of Radiation Oncology, Shandong Cancer Hospital and Institute, Shandong First Medical University and Shandong Academy of Medical Sciences, Jiyan Road 440, Jinan 250117, Shandong, China. ${ }^{3}$ Department of Human Resource, Shandong Cancer Hospital and Institute, Shandong First Medical University and Shandong Academy of Medical Sciences, Jinan 250117, Shandong, China. ${ }^{4}$ Department of Surgical Oncology, Shandong Cancer Hospital and Institute, Shandong First Medical University and Shandong Academy of Medical Sciences, Jinan 250117, Shandong, China.

\section{Received: 5 November 2020 Accepted: 28 April 2021}

Published online: 22 May 2021

\section{References}

1. Bledsoe TJ, Nath SK, Decker RH. Radiation pneumonitis. Clin Chest Med. 2017;38(2):201-8. https://doi.org/10.1016/j.ccm.2016.12.004.

2. Jain V, Berman AT. Radiation Pneumonitis: Old Problem, New Tricks. Cancers (Basel). 2018;10(7):222. https://doi.org/10.3390/cancers10070222.

3. Trott KR, Herrmann T, Kasper M. Target cells in radiation pneumopathy. Int Radiat Oncol Biol Phys. 2004;58(2):463-9. https://doi.org/10.1016/j.jjrobp.2 003.09.045.

4. Liang B, Yan H, Tian Y, Chen X, Yan L, Zhang T, et al. Dosiomics: extracting 3D spatial features from dose distribution to predict incidence of radiation pneumonitis. Front Oncol. 2019;9:269. https://doi. org/10.3389/fonc.2019.00269.

5. Seppenwoolde Y, De Jaeger K, Lebesque JV. In regard to Tsujino et al. predictive value of dose-volume histogram parameters for predicting radiation pneumonitis after concurrent chemoradiation for lung cancer. IJROBP 2003;55:110-115. Int J Radiat Oncol Biol Phys. 2003;56(4):1208-9; author reply 1209. https://doi.org/10.1016/S0360-3016(03)00345-6.

6. Kong FM, Hayman JA, Griffith KA, Kalemkerian GP, Arenberg D, Lyons S, et al. Final toxicity results of a radiation-dose escalation study in patients with non-small-cell lung cancer (NSCLC): predictors for radiation pneumonitis and fibrosis. Int J Radiat Oncol Biol Phys. 2006;65(4):1075-86. https://doi.org/10.1016/j.jijobp.2006.01.051.

7. Luna JM, Chao HH, Diffenderfer ES, Valdes G, Chinniah C, Ma G, et al. Predicting radiation pneumonitis in locally advanced stage II-III non-small cell lung cancer using machine learning. Radiother Oncol. 2019;133:106-12. https://doi.org/10.1016/j.radonc.2019.01.003.

8. Wang W, Xu Y, Schipper M, Matuszak MM, Ritter T, Cao Y, et al. Effect of normal lung definition on lung dosimetry and lung toxicity prediction in radiation therapy treatment planning. Int J Radiat Oncol Biol Phys. 2013; 86(5):956-63. https://doi.org/10.1016/j.jijrobp.2013.05.003.

9. Giuliani ME, Lindsay PE, Kwan JYY, Sun A, Bezjak A, le LW, et al. Correlation of dosimetric and clinical factors with the development of esophagitis and radiation pneumonitis in patients with limited-stage small-cell lung carcinoma. Clin Lung Cancer. 2015;16(3):216-20. https://doi.org/10.1016/j. cllc.2014.11.008.

10. $Y u$ H, Wu H, Wang W, Jolly S, Jin JY, Hu C, et al. Machine learning to build and validate a model for radiation pneumonitis prediction in patients with non-small cell lung Cancer. Clin Cancer Res. 2019;25(14):4343-50. https://doi. org/10.1158/1078-0432.CCR-18-1084.

11. Schallenkamp JM, Miller RC, Brinkmann DH, Foote T, Garces YI. Incidence of radiation pneumonitis after thoracic irradiation: dose-volume correlates. Int J Radiat Oncol Biol Phys. 2007;67(2):410-6. https://doi.org/10.1016/j.jijobp.2 006.09.030.

12. Inoue T, Fujishima S, Ikeda E, Yoshie O, Tsukamoto N, Aiso S, et al. CCL22 and CCL17 in rat radiation pneumonitis and in human idiopathic pulmonary fibrosis. Eur Respir J. 2004;24(1):49-56. https://doi.org/10.1183/ 09031936.04.00110203.

13. Shinde A, Yang D, Frankel P, Liu A, Han C, del Vecchio B, et al. Radiationrelated toxicities using organ sparing Total marrow irradiation transplant conditioning regimens. Int J Radiat Oncol Biol Phys. 2019;105(5):1025-33. https://doi.org/10.1016/j.jijrobp.2019.08.010.

14. Xu L, Jiang J, Li Y, Zhang L, Li Z, Xian J, et al. Genetic variants of SP-D confe susceptibility to radiation pneumonitis in lung cancer patients undergoing thoracic radiation therapy. Cancer Med. 2019;8(5):2599-611. https://doi.org/1 $0.1002 /$ cam4.2088

15. Tang $Y$, Yang L, Qin W, Yi M', Liu B, Yuan X'. Validation study of the association between genetic variant of $I L 4$ and severe radiation pneumonitis in lung cancer patients treated with radiation therapy. Radiother Oncol. 2019;141:8694. https://doi.org/10.1016/j.radonc.2019.09.002.

16. Tang Y, Yang L, Qin W, Yi M'X, Liu B, Yuan X'L. Impact of genetic variant of HIPK2 on the risk of severe radiation pneumonitis in lung cancer patients treated with radiation therapy. Radiat Oncol. 2020;15(1):9. https://doi.org/1 0.1186/s13014-019-1456-0.

17. Barriger RB, Fakiris AJ, Hanna N, Yu M, Mantravadi P, McGarry RC. Dosevolume analysis of radiation pneumonitis in non-small-cell lung cancer patients treated with concurrent cisplatinum and etoposide with or without consolidation docetaxel. Int J Radiat Oncol Biol Phys. 2010;78(5):1381-6. https://doi.org/10.1016/j.ijrobp.2009.09.030.

18. Graves PR, Siddiqui F, Anscher MS, Movsas B. Radiation pulmonary toxicity: from mechanisms to management. Semin Radiat Oncol. 2010;20(3):201-7. https://doi.org/10.1016/j.semradonc.2010.01.010

19. National Cancer Institute. Common Terminology Criteria for Adverse Events (CTCAE) version 5.0 2017. Available from: https://ctep.cancer.gov/ protocolDevelopment/electronic_applications/ctc.htm.

20. Rodrigues G, Lock M, D'Souza D, Yu E, van Dyk J. Prediction of radiation pneumonitis by dose - volume histogram parameters in lung cancer--a systematic review. Radiother Oncol. 2004;71(2):127-38. https://doi.org/10.101 6/j.radonc.2004.02.015.

21. Palma DA, Senan S, Tsujino K, Barriger RB, Rengan R, Moreno M, et al. Predicting radiation pneumonitis after chemoradiation therapy for lung cancer: an international individual patient data meta-analysis. Int J Radiat Oncol Biol Phys. 2013;85(2):444-50. https://doi.org/10.1016/j.jjrobp.2012.04. 043.

22. Tonison JJ, Fischer SG, Viehrig M, Welz S, Boeke S, Zwirner K, et al. Radiation pneumonitis after intensity-modulated radiotherapy for esophageal Cancer: institutional data and a systematic review. Sci Rep. 2019;9(1):2255. https:// doi.org/10.1038/s41598-018-38414-5.

23. Wang L, Liang S, Li C, Sun X, Pang L, Meng X, et al. A novel Nomogram and risk classification system predicting radiation pneumonitis in patients with esophageal Cancer receiving radiation therapy. Int J Radiat Oncol Biol Phys. 2019;105(5):1074-85. https://doi.org/10.1016/j.jirobp.2019.08.024. 
24. Murro D, Jakate S. Radiation esophagitis. Arch Pathol Lab Med. 2015;139(6): 827-30. https://doi.org/10.5858/arpa.2014-0111-RS.

25. Jeffrey B, Benjamin M. Radiation esophagitis: predictive factors and preventive strategies. Semin Radiat Oncol. 2004 Oct; 14(4):280-6.

26. Antonadou D, Coliarakis N, Synodinou M, Athanassiou H, Kouveli A, Verigos C, et al. Randomized phase III trial of radiation treatment + /- amifostine in patients with advanced-stage lung cancer. Int J Radiat Oncol Biol Phys. 2001;51(4):915-22. https://doi.org/10.1016/S0360-3016(01)01713-8.

27. Hawkins PG, Boonstra PS, Hobson ST, Hayman JA, ten Haken RK, Matuszak $\mathrm{MM}$, et al. Prediction of radiation esophagitis in non-small cell lung Cancer using clinical factors, Dosimetric parameters, and pretreatment cytokine levels. Transl Oncol. 2018;11(1):102-8. https://doi.org/10.1016/j.tranon.201 7.11.005.

28. Wada K, et al. Predictors of acute radiation esophagitis in non-small cell lung Cancer patients treated with accelerated Hyperfractionated Chemoradiotherapy. Anticancer Res. 2019;39(1):491-7. https://doi.org/10.21 873/anticanres.13139.

29. Luo Y, McShan DL, Matuszak MM, Ray D, Lawrence TS, Jolly S, et al. A multiobjective Bayesian networks approach for joint prediction of tumor local control and radiation pneumonitis in nonsmall-cell lung cancer (NSCL C) for response-adapted radiotherapy. Med Phys. 2018;45(8):3980-95. https://doi.org/10.1002/mp.13029.

30. Wang S, Campbell J, Stenmark MH, Stanton P, Zhao J, Matuszak MM, et al. A model combining age, equivalent uniform dose and IL-8 may predict radiation esophagitis in patients with non-small cell lung cancer. Radiother Oncol. 2018;126(3):506-10. https://doi.org/10.1016/j.radonc.2017.12.026.

\section{Publisher's Note}

Springer Nature remains neutral with regard to jurisdictional claims in published maps and institutional affiliations.

Ready to submit your research? Choose BMC and benefit from:

- fast, convenient online submission

- thorough peer review by experienced researchers in your field

- rapid publication on acceptance

- support for research data, including large and complex data types

- gold Open Access which fosters wider collaboration and increased citations

- maximum visibility for your research: over $100 \mathrm{M}$ website views per year

At $\mathrm{BMC}$, research is always in progress.

Learn more biomedcentral.com/submissions 